\title{
Analytical Procedure Accuracy
}

National Cancer Institute

\section{Source}

National Cancer Institute. Analytical Procedure Accuracy. NCI Thesaurus. Code C134242.

The closeness of agreement between the value which is accepted either as a conventional true value or an accepted reference value and the value found. 